\title{
Impact of Acupuncture on Sleep and Comorbid Symptoms for Chronic Insomnia: A Randomized Clinical Trial
}

\author{
Cong Wang ${ }^{1} * *$ \\ Wen-lin $X u^{l}{ }^{1} *$ \\ Guan-wu $\mathrm{Li}^{2}$ \\ Cong $\mathrm{Fu}^{\prime}$ \\ Jin-jin $L i^{\prime}$ \\ Jing Wang (D) \\ Xin-yu Chen ${ }^{3}$ \\ Zhen Liu' \\ Yun-fei Chen' \\ 'Department of Acupuncture and \\ Moxibustion, Yueyang Hospital of \\ Integrated Traditional Chinese and \\ Western Medicine, Shanghai University of \\ Traditional Chinese Medicine, Shanghai, \\ People's Republic of China; ${ }^{2}$ Department \\ of Radiology, Yueyang Hospital of \\ Integrated Traditional Chinese and \\ Western Medicine, Shanghai University of \\ Traditional Chinese Medicine, Shanghai, \\ People's Republic of China; ${ }^{3}$ Acupuncture \\ and Tuina Academy, Beijing University of \\ Chinese Medicine Dongfang College, \\ Hebei, People's Republic of China
}

*These authors contributed equally to this work
Correspondence: Yun-fei Chen; Zhen Liu Department of Acupuncture and Moxibustion, Yueyang Hospital of Integrated Traditional Chinese and

Western Medicine, Shanghai University of Traditional Chinese Medicine, No. II0 Ganhe Road, Hongkou District, Shanghai, 200437, People's Republic of China

$\mathrm{Tel} / \mathrm{Fax}+86-2 \mathrm{I}-65 \mathrm{I} 62628$

Email icyf1968@I63.com;

liuzhen8918@163.com
Study Objectives: To evaluate the efficacy and safety of acupuncture at HT 7 (Shenmen) and KI 7 (Fuliu) on sleep and comorbid symptoms for chronic insomnia.

Methods and Design: A randomized, single-blind, parallel and sham-controlled trial consisted of an acupuncture group $(n=41)$ and a sham acupuncture group $(n=41)$. Setting: a tertiary hospital of integrated Chinese and Western medicine. Participants: 82 subjects with chronic insomnia based on the International Classification of Sleep Disorders, Third Edition (ICSD-3). Interventions: a 10-session acupuncture treatment at bilateral HT 7 and KI 7 or sham acupoints with shallow needling was performed over 3 weeks. Measurements: the Pittsburgh sleep quality index (PSQI) and insomnia severity index (ISI) were evaluated at baseline, posttreatment, and at two follow-ups as the primary outcome measures. Polysomnography (PSG) on two consecutive nights, the Beck anxiety inventory (BAI), the Beck depression inventory (BDI) fatigue severity scale (FSS) and the Epworth sleepiness scale (ESS) were evaluated at baseline and posttreatment as the secondary outcome measures.

Results: After the treatments, PSQI scores decreased by 5.04 in the acupuncture group and 2.92 in the sham acupuncture group. ISI scores decreased by 7.65 in the acupuncture group and 5.05 in the sham acupuncture group. The between-group differences in the primary outcome measures posttreatment were statistically significant. However, no differences were found between the two groups during the two follow-ups. Regarding the PSG data, there were significantly lower levels of sleep onset latency (SOL), a lower percentage of sleep stage N1 and a higher percentage of sleep stage N3 in the acupuncture group than in the sham acupuncture group. After treatment, there were lower levels of comorbid symptoms (BAI, BDI, FSS and ESS) in both groups. However, no significant differences were observed between the groups.

Conclusion: Acupuncture at HT 7 and KI 7 is an effective and safe nonpharmacologic intervention option for chronic insomnia.

Clinical Trial Registration: The study was registered at the Chinese Clinical Trial Registry, registration ID: ChiCTR1900023787, China.

Keywords: acupuncture, chronic insomnia, sleep and comorbid symptoms, randomized clinical trial

\section{Plain Language Summary}

Acupuncture has been used to treat chronic insomnia for years, but no study has focused on the comprehensive evaluation of acupuncture on sleep (subjective and objective) and comorbid symptoms. This was the first randomized controlled clinical trial to study the 
therapeutic effect of acupuncture at a pair of acupoints on insomnia. This randomized controlled clinical study showed that, compared with sham acupuncture, the streamlined and optimized acupuncture programme could improve subjective and objective sleep measurements of subjects with chronic insomnia, but there was no significant improvement in comorbid symptoms. This study led to a more comprehensive understanding of this nonpharmaceutical intervention. However, the optimal plan and mechanism of acupuncture treatment for chronic insomnia need to be further determined.

\section{Introduction}

Insomnia is a frequently occurring clinical disease that affects people's lives in our society. The incidence of chronic insomnia has increased year by year. ${ }^{1}$ According to the diagnostic criteria of the Diagnostic and Statistical Manual of Mental Disorders-4th (DSM-IV), the incidence rate of chronic insomnia in Chinese adults is $19.6 \%{ }^{2}$ Long-term chronic insomnia can cause serious health problems and many other chronic diseases such as diabetes, ${ }^{3}$ obesity, ${ }^{4}$ cardiovascular disease, ${ }^{5}$ chronic kidney disease, ${ }^{6}$ and hypertension. ${ }^{7}$ The most direct harm is effects on quality of life. ${ }^{8}$ Furthermore, patients with chronic insomnia usually have mood disorders, ${ }^{9,10}$ and chronic insomnia is often accompanied by daytime dysfunction. ${ }^{11}$ All these factors can deteriorate the severity of chronic insomnia and contribute to the difficulty of treatment, which requires clinical attention and a search for simpler and faster treatment options.

At present, cognitive behavioural therapy for insomnia (CBT-I) and other psychotherapeutic approaches are generally accepted internationally as the primary recommended treatment for chronic insomnia disorder. ${ }^{12,13}$ However, due to its time-intensive nature and because physicians need much professional training to conduct them, ${ }^{14}$ these methods are not fully implemented in China. Although pharmacologic treatments have the advantages of being effective and fast, there are many side effects of sedative-hypnotic medicine, such as cognitive impairment, hangover effects, and drug addiction. ${ }^{15,16}$ Therefore, more patients with chronic insomnia seek complementary and alternative therapies, such as acupuncture, especially among Chinese people. ${ }^{17}$ Studies have shown that acupuncture can improve sleep quality and psychological health. ${ }^{18,19}$ An earlier Cochrane review showed that, when compared with untreated subjects and those receiving placebo acupuncture, acupuncture can significantly improve sleep quality among subjects with insomnia. $^{20}$ Our previous study also indicated that acupuncture has better clinical efficacy and safety in perimenopausal insomnia than sham acupuncture. ${ }^{21}$

However, most of the previous studies on acupuncture for insomnia have some general disadvantages, such as an unreasonable control group, no randomization, no blinding method, and no objective indicators for sleep evaluation. ${ }^{22-24}$ In addition, the existing studies generally lack the guidance of traditional Chinese medicine (TCM) theory, ${ }^{25,26}$ and the selection of acupoints is highly variable, ${ }^{27}$ which increases the difficulty of implementation and increases subject pain. Most of the studies on acupuncture for insomnia did not use comprehensive indicators to evaluate sleep and related symptoms. However, the Recommendations for a Standard Research Assessment of Insomnia highlight that evaluating and reporting the diagnosis of insomnia and its comorbid symptoms, sleep symptoms, and waking correlates and consequences of insomnia (ie, fatigue, depression, anxiety, quality of life and function) are the basic elements for treatment efficacy and effectiveness studies. ${ }^{28}$ More studies are needed to investigate the subjective and objective indicators of acupuncture for improving sleep and explore the secondary benefits of treating insomnia, such as reductions in depression, anxiety, fatigue, and daytime sleepiness.

According to TCM theory, the main causes of chronic insomnia are Yin deficiency leading to heart fire and dysregulated coordination between the heart and the kidney. ${ }^{29}$ Therefore, we chose acupoints based on disease differentiation and special acupoint combinations to nourish Yin, calm the mind, and regulate mental activity. HT 7 (Shenmen) and KI 7 (Fuliu) are adopted as the points to nourish Yin, and they are especially used to nourish Yin of the liver and kidney and decrease heart fire. ${ }^{30,31}$ According to the theory of TCM, acupuncture at this pair of acupoints can solve sleep problems and related symptoms of insomnia. There are no reports on the treatment of insomnia through these two acupoints in the existing literature. This was the first randomized controlled clinical trial to study the therapeutic effect of acupuncture at HT 7 and KI 7 on insomnia. The objective was to explore the efficacy and safety of acupuncture at HT 7 and KI 7 on sleep and comorbid symptoms for chronic insomnia in shamcontrolled, subject- and assessor-blind, randomized controlled trials.

\section{Methods}

\section{Study Design}

This was a randomized, single-blind, parallel and shamcontrolled trial. This study followed the Consolidated 
Standards of Reporting Trials (CONSORT) ${ }^{32}$ and Standards for Reporting Interventions in Clinical Trials of Acupuncture (STRICTA) ${ }^{33}$ guidelines for designing and reporting controlled trials. The trial was conducted in accordance with the Declaration of Helsinki and was approved by the Institutional Review Board of Yueyang Hospital of Integrated Traditional Chinese and Western Medicine, Shanghai University of Traditional Chinese Medicine (Shanghai, China).

\section{Participants}

The participants were patients with chronic insomnia who came from the outpatient department of the Department of Acupuncture and Moxibustion, Yueyang Hospital of Integrated Traditional Chinese and Western Medicine, from June 2019 to January 2020. In addition, the study recruited subjects through community lectures and recruitment advertisements. This study followed the principle of voluntary participation and required the signing of an informed consent document before enrolment. All those who wanted to participate in the study called the study coordinator, and we conducted a preliminary clinical interview.

The diagnosis of chronic insomnia was made with clinical interviews according to the American Academy of Sleep Medicine International Classification of Sleep Disorders, Third Edition (ICSD-3). ${ }^{34}$ The inclusion criteria were as follows: (1) 18 to 75 years old. (2) In compliance with the diagnostic criteria for chronic insomnia in ICSD-3. (3) In compliance with the TCM diagnostic criteria for insomnia, TCM syndrome of dysregulated coordination between the heart and the kidney. (4) Consistent administration of sedative-hypnotic drugs for more than 3 months before entering the study or not taking such drugs. (5) A total score higher than five on the Pittsburgh Sleep Quality Index (PSQI) questionnaire. ${ }^{35}$ (6) Patients voluntarily participated in the study and signed informed consent forms.

All subjects who wanted to participate were given an outpatient interview to determine whether they met the inclusion criteria. The interviews were conducted by experienced clinicians in the form of semistructured interview questionnaires. The interview questionnaire included the cause of insomnia, the onset process, the main sleep complaints at the current time, the treatment process, the state before going to bed, the sleep-wake cycle, other sleep-related symptoms, mental states, and daytime symptoms. ${ }^{36}$ Individuals on stable doses of sedative-hypnotic medications were allowed to remain on them during the study. In addition, all subjects completed a one-week sleep diary to establish sleep patterns before the clinical interview. ${ }^{37}$

The exclusion criteria were as follows: (1) severe hepatic or renal function damage, haematological disease, respiratory disease, or diagnosis of one or more mental disorders according to the Diagnostic and Statistical Manual of Mental Disorders (DSM-V). ${ }^{38}$ (2) semistructured interviews determined that subjects had sleep disorders other than chronic insomnia. (3) Infectious diseases such as infectious hepatitis, tuberculosis, AIDS and syphilis. (4) Severe digestive system diseases or severe malnutrition. (5) Pregnant or lactating. (6) Severe trauma with no cure or other contraindications to acupuncture, such as allergic constitution or severe dermatosis. (7) An Apnoea-Hypopnea Index $>10$ or periodic limb movements index during sleep associated with $>15$ arousals per hour on diagnostic PSG. ${ }^{21,39}$ (8) Participation in other clinical trials in the last 3 months.

\section{Intervention}

The acupuncturist had received a master's degree in acupuncture and tui na, had a doctor qualification certificate, and had more than 3 years of acupuncture clinical work experience. All the research assistants received 3 days of training before the start of the study.

Subjects in the acupuncture group were placed in a supine position. The acupuncturist selected two acupoints, HT 7 and KI 7. These acupoints were selected based on a previous systematic review ${ }^{40,41}$ and our clinical experience summary and because the use of only two points can streamline the treatment and alleviate patient pain. The needles used were disposable, sterile, stainlesssteel acupuncture needles with a length of $40 \mathrm{~mm}$ and a diameter of $0.25 \mathrm{~mm}$ (Andy, Guizhou, China). The acupuncturist treated each acupoint bilaterally, and the depth of insertion was 10-20 $\mathrm{mm}$. The acupuncturist selected the depth according to the subject's body type (high, short, fat and thin). After penetration, the acupuncturist lifted and twisted the needles to induce the subject's sensation of "De qi", and the needle was left for 20 minutes. $^{42}$ "De qi" refers to manipulation of the needle by the acupuncturist (extract, intercalate, twist, and rotate) after the needle has been inserted into the acupoints to a certain depth so that the acupoints can be induced by the meridians. The patient has a conscious response, such as soreness, numbness, or tenderness. According to TCM, this is the key to the curative effect of acupuncture. ${ }^{43}$ 
Subjects in the sham acupuncture group were treated with shallow needling of sham acupoints. Due to its convenience, this control group setting method has been widely used in acupuncture clinical research, especially for the purpose of exploring acupoint-specific effects among the Chinese population. ${ }^{44,45}$ Sham HT 7 was 2 cun $(\approx 40 \mathrm{~mm})$ lateral to HT 7, and sham KI 7 was 2 cun ( $\approx 40 \mathrm{~mm})$ lateral to KI 7 (Figure 1). The purpose of choosing the sham points in this way is explained in previous similar clinical studies; ${ }^{45}$ this approach avoids the true acupoints and meridian routes. Choosing sham points close to the true acupoints was conducive to blinding. The acupuncturist took the sham points on both sides and performed needling at a depth of 2-4 mm, sufficient only to penetrate the skin. The acupuncturist did not manipulate the needles and did not cause "De qi". The parameters of the acupuncture needles were identical to those used in the acupuncture group. Subjects in the two groups received the same treatment, which included 3 times a week for a total of 10 treatments. Each subject experienced screening, treatment, and a follow-up period of approximately 8 weeks, and the treatment phase lasted approximately 3.5 weeks. All subjects were treated equally to prevent them from perceiving differences. They were told about the methods of acupuncture as follows:

In our study, different types of acupuncture treatments will be compared. One is ordinary acupuncture, and the other is related to the positive results in previous clinical studies. ${ }^{46}$

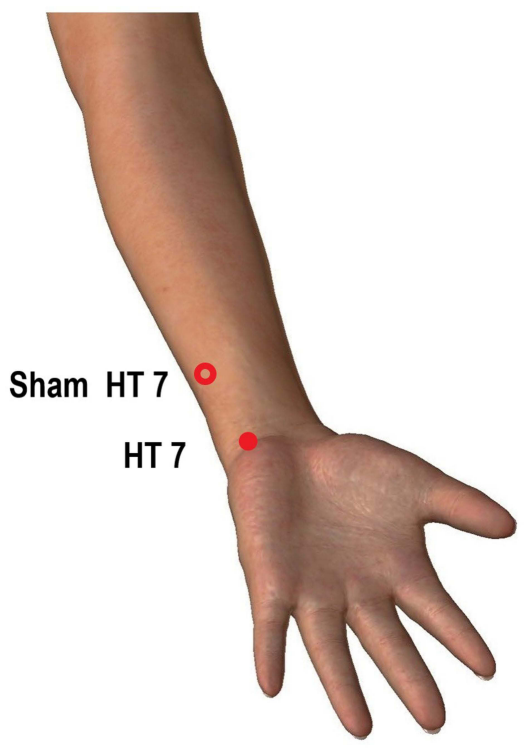

\section{Measures}

\section{Primary Outcome Measures}

Pittsburgh Sleep Quality Index (PSQI): The PSQI contains 19 self-assessed items and 5 other-rated items. The selfassessed items evaluate duration of sleep, sleep onset latency, sleep efficiency, sleep disturbance, need for medications to sleep, daytime dysfunction, and overall sleep quality. The total score is $0-21$. A higher score indicates a worse quality of sleep. ${ }^{35}$ The PSQI is widely used to evaluate sleep dysfunction, ${ }^{47}$ and it is more inclined to evaluate the subject's sleep state during the workday. ${ }^{48}$ A total score $>5$ indicates that the subjects have poor sleep quality and has significance for clinical treatment. ${ }^{49}$ The Chinese version of the PSQI has high reliability and validity for the Chinese population and has been used as an effective tool for sleep screening in clinical and scientific research. ${ }^{50,51}$ This study evaluated the PSQI at baseline, posttreatment, one-week follow-up and one-month follow-up.

Insomnia Severity Scale (ISI): The ISI mainly evaluates the nature and severity of insomnia and its impact on daytime function. The scale consists of 7 evaluation items, and the total score ranges from 0-28 points. A higher score indicates more severe insomnia. ${ }^{52}$ The ISI is mainly used to screen for insomnia and to evaluate treatment effects in clinical research. ${ }^{53}$ A total score of 8-14 indicates subclinical insomnia, and a total score $>14$ indicates

Figure I Acupoints for the acupuncture and sham acupuncture groups.

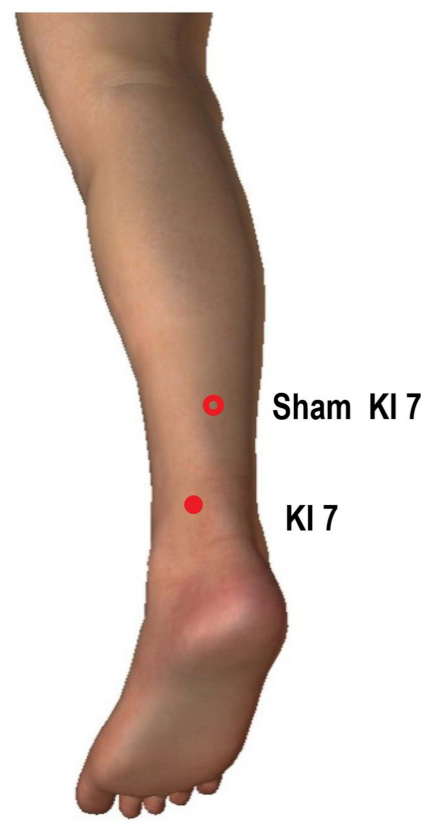


clinically significant insomnia. A reduction in the ISI score by 8 points or more reflects a significant treatment effect in clinical evaluations. ISI has good reliability and validity. ${ }^{54}$ The Chinese version has high reliability and validity for evaluating insomnia among the Chinese population. ${ }^{55}$ This study evaluated ISI at baseline, posttreatment, one-week follow-up and one-month follow-up.

\section{Secondary Outcome Measures}

Polysomnography (PSG): PSG was used as the objective sleep evaluation index. The PSG (NIHON KOHDEN, Japan) used in this study was composed of multiple quantitative parameters. The collection indicators included electroencephalogram (EEG), mentalis electromyography $(\mathrm{EMG})$, bilateral electrooculography $(\mathrm{ECoG})$, oral/nasal airflow, and bilateral anterior tibial EMG. In this study, PSG was used for two consecutive nights to eliminate the "first night effect". ${ }^{56}$ PSG monitoring started from the usual bedtime to the next morning, and a total of 8.0 hours were recorded. The efficacy evaluation of this study was based on EEG. The sleep stages and parameters were manually scored based on the American Academy of Sleep Medicine Manual for the Scoring of Sleep and Associated Events. ${ }^{57}$ The objective sleep evaluation parameters used in this study included the following: total sleep duration (TST); sleep onset latency (lights out to first epoch of any sleep, SOL); REM sleep latency (REMsl); wake after sleep onset (WASO, SOL minus TST); sleep efficiency (SE, TST/total recording time $\times 100 \%$ ); arousal index (Arl; number of arousals $\times 60 / \mathrm{TST}$ ); and percent time in each stage (length of each sleep stage $/ \mathrm{TST} \times 100 \%$ ). In this study, during the screening period and at posttreatment, two consecutive monitorings were performed, but only the second result for each timepoint was used as the efficacy evaluation index.

Beck Anxiety Inventory (BAI): The BAI was developed by Beck et al in $1988^{58}$ and contains a total of 21 anxiety symptom items. It has been used as an evaluation index to evaluate the anxiety state of the subject within the past week. Higher scores correspond to more serious anxiety. Generally, $\mathrm{BAI} \geq 45$ is used as the criterion to indicate anxiety. The scale is simple in content, easy to administer, and easily understood. The Chinese version has good reliability and validity and is commonly used for the evaluation of anxiety symptoms in China. ${ }^{59,60}$ This study evaluated it at baseline and posttreatment.

Beck Depression Inventory (BDI): The BDI was developed by Beck et al according to the diagnostic criteria for depression in the DSM-IV. ${ }^{61}$ Beck et al formulated the first version of the BDI in 1961 and restructured it for the second version, which is now generally used. The BDI has a total of 21 self-evaluated depression-related items that can evaluate depression in the past 7 days. The higher the total score, the more severe the depression. A total score of $0-13$ indicates no depression, 14-19 indicates mild depression, and 29-63 indicates severe depression. The Chinese version of the BDI has good validity and reliability. ${ }^{62,63}$ This study evaluated it at baseline and posttreatment.

Fatigue Severity Scale (FSS): FSS was proposed and formulated by Krupp et al in $1989 .{ }^{64}$ The FSS scale is simple and clear. It consists of only 9 self-evaluation items. Subjects scored themselves on the corresponding symptoms. Higher total scores indicate more fatigue. Studies have shown that the FSS score of patients with insomnia is higher than 5.5, which means that there is a high level of daytime fatigue among patients with insomnia. ${ }^{65}$ The Chinese version of the FSS has good validity and reliability. ${ }^{66}$ This study evaluated the FSS at baseline and posttreatment.

Epworth Sleepiness Scale (ESS): The ESS is a relatively simple and internationally recognized sleepiness assessment scale. ${ }^{67}$ It has a total score of 24 points. The higher the score is, the more severe the daytime sleepiness. This scale is generally used to screen and evaluate the hypopnea status of patients with obstructive sleep apnoea-hypopnea syndrome (OSAHS), but studies have shown that the ESS scale can be used to screen and diagnose other sleep disorders. ${ }^{68}$ The Chinese version of the ESS has good validity and reliability. ${ }^{69}$ This study used the ESS to assess the daytime sleepiness of subjects at baseline and posttreatment.

\section{Safety Assessments}

Two types of safety assessments were performed: evaluation of changes in routine blood tests, liver function, and kidney function after the 10 intervention sessions and records of adverse events during the entire study. The record of adverse events in this study was conducted using the Adverse Event Record Form. Subjects were required to fill in the list of adverse events during the study, including the time point, severity, measures taken, whether the event was related to the treatment and prognosis. During the assessment phase, researchers assessed the possible relationship between adverse events and the study treatment, as well as medications taken concurrently. Adverse events include all adverse reactions that are 
definitely related to treatment, most likely related to treatment, and likely related to treatment.

\section{Sample Size}

The required sample size was estimated based on the change in PSQI scores. According to previous research by our team, ${ }^{21}$ sham acupuncture can reduce the PSQI to 14.76 after 10 interventions, with an SD of 3.35. This study hypothesized that acupuncture at HT 7 and KI 7 for insomnia would reduce the PSQI by 2.70 points more than sham acupuncture. According to the needs of this study, we made $\alpha=0.05$ and $1-\beta=0.90$, according to the formula: ${ }^{70}$

$$
\mathrm{n}=2\left[\frac{\left(u_{\alpha}+u_{\beta}\right)}{\delta / \sigma}\right]^{2}+\frac{1}{4} u_{\alpha}{ }^{2}
$$

A two-sided test was performed. Through the query, $u_{0.05 / 2}=1.96, u_{0.1}=1.282$, and calculated $\mathrm{n}=33.32 \approx 34$. In addition, considering the loss factor (according to a $20 \%$ loss rate), the final sample size was 41 subjects in each group, for 82 subjects in total.

\section{Randomization and Blinding}

Specialized statisticians who were independent of the research group generated 82 random numbers in SPSS 25.0 and then arranged these numbers in order from small to large. Numbers $1-41$ were artificially specified as the acupuncture group, and $42-82$ were specified as the sham acupuncture group. The random numbers are reproducible (random seed number: 20190520). Subjects entered the acupuncture group or the sham acupuncture group according to randomly assigned numbers at a ratio of 1:1 after signing the informed consent form. The random number and treatment plan corresponding to each subject were printed on a card and stored in an opaque envelope. Each subject who signed the informed consent form was given an envelope with their name, which was handed over to the acupuncturist during the treatment. The acupuncturist checked the envelope and removed the treatment card to view the treatment plan.

During the study, the generation of the random numbers list, subject recruitment, acupuncture treatment, outcome measure assessment and follow-up were performed independently by different researchers. Only the outside assistant and the acupuncturist were aware of the allocation. Participants and other relevant researchers were blinded to the allocation.

\section{Statistical Analysis}

The statistics and analysis of all data were performed by two analytical researchers independent of the trial. Intention-to-treat (ITT) was used as the evaluation method to evaluate the results of the study. Statistical analysis of the data was performed using SPSS 25.0. The measurement data conformed to a normal distribution, and homogeneous variances were determined using the mean \pm standard deviation to describe the central tendency and the degree of dispersion. The primary outcome assessments were analysed using a mixed-effect model for repeated measures (MMRM) with patients as a randomeffect factor, treatment group, age, gender, time and comorbidities as the fixed variable, patients as the random variable, random effects including intercept, baseline as a covariable, age*group, gender*group, time*group and comorbidities*group as interaction effect using a firstorder ante dependence covariance matrix. The comparison between groups used the independent sample $t$ test, and the comparison of multiple time points within groups used repeated measurement data combined with multivariate variance analysis. For measurement data that did not conform to the normal distribution, the median, minimum, and maximum were given. The between-group comparisons used the Mann-Whitney $U$-test, and the within-group comparisons used the Friedman (F) test. Enumeration data were expressed as frequency and composition rate. For binary or multicategory nonranked data, comparisons between groups were performed by the $\chi 2$ test. If the data were ranked as ordinal data, the comparison between groups was performed by the Mann-Whitney $U$-test.

All statistical analyses used two-sided tests, and $P$ values of $\leq .05$ were considered statistically significant.

\section{Results}

\section{Subject Characteristics}

A total of 91 subjects were recruited, of whom 9 were excluded during screening. Eighty-two subjects with chronic insomnia meeting the criteria were included in this study and were randomly assigned to the acupuncture group or the sham acupuncture group. Among the subjects, 4 withdrew for personal reasons; 1 withdrew due to poor results; 1 withdrew due to adverse reactions; and 1 completed the treatment but did not undergo posttreatment evaluation. Therefore, a total of 75 eligible subjects completed the treatment and follow-up stage, including 37 in 
the acupuncture group and 38 in the sham acupuncture group (Figure 2).

Table 1 presents the demographic characteristics of the subjects. Subjects' ages ranged from 25 to 73 (median 58 years), $23(28.0 \%)$ were male, and 59 (72.0\%) were female. The majority of the subjects were normal weight $(\mathrm{n}=68,82.9 \%)$. The subjects had a longer course of insomnia (median 9.5 years). Approximately 53.7\% of the subjects were taking sedative-hypnotic drugs. There were no differences in the demographics between the two groups. Baseline clinical characteristics were also balanced between the two groups (Tables 2 and 3).

\section{Efficacy}

\section{Primary Outcome Assessments}

The mixed-effect model analysis revealed that there were significant effects for the time $\times$ treatment interaction on the PSQI $(F=4.756, P=0.004)$ (Table 2 and Figure 3), which means that the change trend of the PSQI over time was different due to the different groups. The mixedeffect model analysis also showed significant effects for the time $\times$ treatment interaction on the ISI $(F=4.130$,
$P=0.008$ ) (Table 2 and Figure 4), which means that the change trend of the ISI over time was different due to different groups. There were improvements in PSQI and ISI scores after either acupuncture or sham acupuncture treatment (Table 3). PSQI scores decreased from 14.78 to 9.74 in the acupuncture group and from 14.61 to 11.69 in the sham acupuncture group. After treatment, the difference between groups was statistically significant $(F=$ $-2.537, P=0.013)$. ISI scores at baseline were 18.78 for the acupuncture group and 20.49 for the sham acupuncture group. The scores decreased to 11.13 for the acupuncture group and 15.44 for the sham acupuncture group. After treatment, the difference between groups was statistically significant $(F=-3.796, P<0.001)$. Although the PSQI and ISI scores were lower than at baseline at the one-week follow-up and one-month follow-up, no differences were observed between groups ( $P>0.05$ for all comparisons).

\section{Secondary Outcome Assessments PSG Assessments}

The results of PSG measurements are summarized in Table 4. After treatment, there were significantly lower levels of SOL $[15.5(10.0,18.5)$ vs $18.5(11.5,31.0), P=0.039)$ in the

25 were excluded

- $n=3$ Age $>75$ years old

- $n=4$ Unwilling to undergo laboratory tests

- $n=6$ Diagnosed with other sleep disorders

- $\mathrm{n}=2$ Diagnosed with psychiatric disorders

- $n=6$ Disagreed with the research plan

- $\mathrm{n}=2$ Irregular use sedative-hypnotic drugs

- $\mathrm{n}=2$ Suspected of having obstructive sleep apnea-hypopnea syndrome
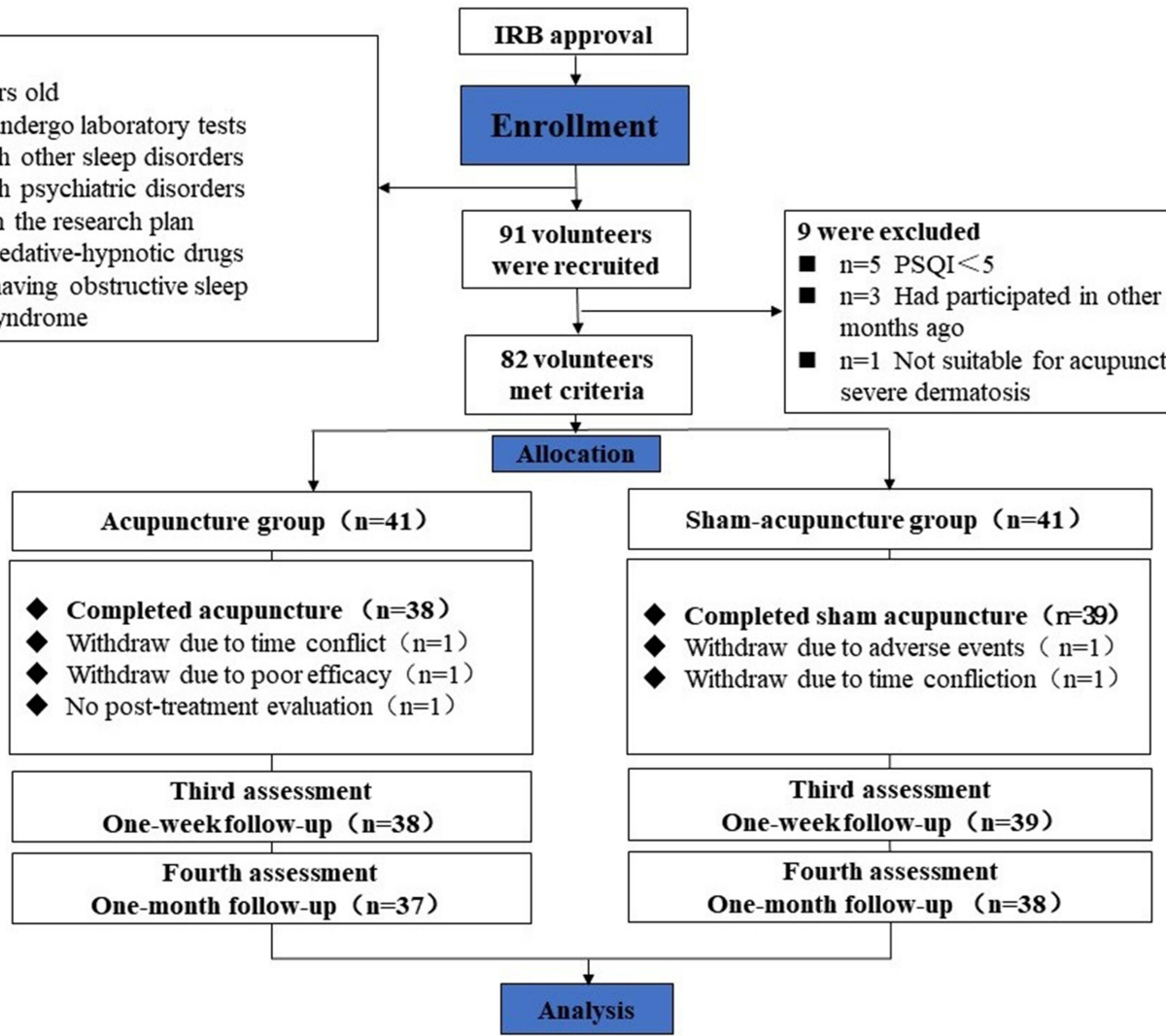

Figure 2 Flow of subjects randomized to acupuncture or sham acupuncture. 
Table I Demographic Characteristics of Subjects

\begin{tabular}{|c|c|c|c|c|c|}
\hline Variable & $\begin{array}{l}\text { Total Sample } \\
(n=82)\end{array}$ & $\begin{array}{l}\text { Acupuncture } \\
(n=4 I)\end{array}$ & $\begin{array}{l}\text { Sham Acupuncture } \\
(n=4 I)\end{array}$ & Zl $\chi^{2}$ Value $^{\mathbf{a}}$ & $P$ \\
\hline Age, y & $58(25,73)$ & $57(25,72)$ & $58(25,73)$ & -0.264 & 0.791 \\
\hline $\begin{array}{l}\text { Gender } \\
\text { Male } \\
\text { Female }\end{array}$ & $\begin{array}{l}- \\
23(28.0 \%) \\
59(72.0 \%)\end{array}$ & $\begin{array}{l}- \\
13(31.7 \%) \\
28(69.3 \%)\end{array}$ & $\begin{array}{l}- \\
10(24.4 \%) \\
31(75.6 \%)\end{array}$ & 0.544 & 0.461 \\
\hline $\begin{array}{l}\text { BMI, } \mathrm{kg} / \mathrm{m}^{2} \\
\text { Under weight } \\
\text { Normal weight } \\
\text { Overweight } \\
\text { Obesity }\end{array}$ & $\begin{array}{l}- \\
2(2.4 \%) \\
68(82.9 \%) \\
10(12.3 \%) \\
2(2.4 \%)\end{array}$ & $\begin{array}{l}- \\
I(2.4 \%) \\
36(87.8 \%) \\
3(7.4 \%) \\
I(2.4 \%)\end{array}$ & $\begin{array}{l}- \\
I(2.4 \%) \\
32(78.0 \%) \\
7(17.2 \%) \\
I(2.4 \%)\end{array}$ & -1.106 & 0.269 \\
\hline $\begin{array}{l}\text { Education } \\
\text { Primary school } \\
\text { Junior high school } \\
\text { High school } \\
\text { College degree } \\
\text { Graduate degree }\end{array}$ & $\begin{array}{l}- \\
3(3.8 \%) \\
11(13.6 \%) \\
20(24.4 \%) \\
40(48.4 \%) \\
8(9.8 \%)\end{array}$ & $\begin{array}{l}- \\
I(2.4 \%) \\
7(17.1 \%) \\
9(21.9 \%) \\
20(48.8 \%) \\
4(9.8 \%)\end{array}$ & $\begin{array}{l}- \\
2(4.9 \%) \\
4(9.8 \%) \\
11(27.1 \%) \\
20(48.4 \%) \\
4(9.8 \%)\end{array}$ & 1.352 & 0.853 \\
\hline Insomnia duration, y & $9.5(0.3,40.0)$ & $6.0(0.3,40.0)$ & $10.0(0.5,40.0)$ & -1.297 & 0.195 \\
\hline Concomitant diseases & $35(42.7 \%)$ & $18(43.9 \%)$ & $17(41.5 \%)$ & 0.050 & 0.823 \\
\hline Drug allergy & $20(24.4 \%)$ & $12(29.3 \%)$ & $8(19.5 \%)$ & 1.058 & 0.304 \\
\hline $\begin{array}{l}\text { Current hypnotics } \\
\text { Never } \\
\text { Taking one or } \\
\text { more }\end{array}$ & $\begin{array}{l}- \\
38(46.3 \%) \\
44(53.7 \%)\end{array}$ & $\begin{array}{l}- \\
18(43.9 \%) \\
23(56.1 \%)\end{array}$ & $\begin{array}{l}- \\
20(48.8 \%) \\
21(51.2 \%)\end{array}$ & -0.603 & 0.546 \\
\hline
\end{tabular}

Notes: Data are presented as either M (Min, Max) or number (\%). ${ }^{a}$ The measurement data did not conform to normality after the Shapiro-Wilk test. Comparison between the acupuncture and sham acupuncture groups by Mann-Whitney U-test or $\chi^{2}$ test.

Table 2 The Mixed Effect Model Analysis of Primary Outcome Measures

\begin{tabular}{|c|c|c|c|c|c|c|c|c|c|}
\hline & Group & Age & Gender & Time & Comorbidity & $\begin{array}{l}\text { Age } \\
\text { Group* }\end{array}$ & $\begin{array}{l}\text { Gender } \\
\text { Group* }\end{array}$ & $\begin{array}{l}\text { Time } \\
\text { Group * }\end{array}$ & $\begin{array}{l}\text { Comorbidity } \\
\text { Group* }\end{array}$ \\
\hline \multicolumn{10}{|c|}{ PSQI } \\
\hline$F$ & 2.129 & 20.486 & 0.031 & 71.178 & 0.261 & 0.832 & 1.406 & 4.756 & 1.058 \\
\hline$P$ & 0.145 & $<0.001$ & 0.860 & $<0.001$ & 0.609 & 0.362 & 0.236 & 0.004 & 0.304 \\
\hline \multicolumn{10}{|c|}{ ISI } \\
\hline$F$ & 0.440 & 4.222 & 6.264 & 59.901 & 0.005 & 0.038 & 1.682 & 4.130 & 0.534 \\
\hline$P$ & 0.507 & 0.040 & 0.012 & $<0.001$ & 0.946 & 0.845 & 0.195 & 0.008 & 0.465 \\
\hline
\end{tabular}

Notes: Data were analyzed using a mixed-effect model for repeated measures (MMRM). *Interaction.

Abbreviations: PSQI, Pittsburgh sleep quality index; ISI, insomnia severity index.

acupuncture group than in the sham acupuncture group. In terms of sleep architecture, the percentage of sleep stage N1 (19.65 $\pm 5.06 \%$ vs $24.99 \pm 4.60 \%, P=0.015)$ was decreased and that of stage N3 $(25.22 \pm 5.31$ vs $17.88 \pm 4.84, P=0.002)$ was increased significantly in the acupuncture group compared with the sham acupuncture group. However, no significant differences in the percentage of stages N2 or $\mathrm{R}$ were noted between the groups $(P>0.05)$.

\section{Assessment of Comorbid Symptoms}

The results of comorbid symptom assessments are summarized in Table 5. After treatment, there were lower 
Table 3 Primary Outcome Measures from Baseline to 4-Week Follow-Up

\begin{tabular}{|c|c|c|c|c|c|c|c|c|}
\hline Assessments & \multicolumn{2}{|l|}{ Group } & Baseline & Post-Treatment & Follow-Up I & Follow-Up 2 & $\boldsymbol{F}$ & $P$ \\
\hline \multirow[t]{4}{*}{ PSQI score } & Acupuncture $(n=4 I)$ & $\bar{x} \pm s$ & $|4.78 \pm 2.7|$ & $9.74 \pm 3.08$ & $10.66 \pm 3.43$ & $10.5 \mid \pm 3.60$ & 41.694 & $<0.001$ \\
\hline & Sham-acupuncture $(n=4 I)$ & $\bar{x} \pm s$ & $|4.6| \pm 3.1 \mid$ & $11.69 \pm 3.65$ & $11.03 \pm 3.40$ & $10.59 \pm 3.95$ & 32.217 & $<0.001$ \\
\hline & \multirow[t]{2}{*}{ Between-group } & $F$ & 0.265 & -2.537 & -0.472 & -0.075 & - & - \\
\hline & & $P$ & 0.791 & 0.013 & 0.638 & 0.940 & - & - \\
\hline \multirow[t]{4}{*}{ ISI score } & Acupuncture $(n=4 I)$ & $\bar{x} \pm s$ & $18.78 \pm 4.68$ & $11.13 \pm 3.74$ & $12.05 \pm 5.60$ & $12.14 \pm 5.03$ & 43.048 & $<0.001$ \\
\hline & Sham-acupuncture $(n=4 I)$ & $\bar{x} \pm s$ & $20.49 \pm 4.65$ & $15.44 \pm 5.98$ & $14.49 \pm 7.45$ & $13.42 \pm 6.58$ & 34.019 & $<0.001$ \\
\hline & \multirow[t]{2}{*}{ Between-group } & $F$ & -1.657 & -3.796 & -1.624 & -0.949 & - & - \\
\hline & & $P$ & 0.101 & $<0.001$ & 0.109 & 0.346 & - & - \\
\hline
\end{tabular}

Notes: Data are presented as the mean \pm SD. Comparison within-group and between-group by repeated-measures ANOVA.

Abbreviations: PSQI, Pittsburgh sleep quality index; ISI, insomnia severity index.

levels of comorbid symptoms (BAI, BDI, FSS and ESS) in both the acupuncture group and the sham acupuncture group. However, no significant differences were noted between the groups after treatment $(P>0.05)$.

\section{Safety Assessments}

The main observation and recording indicators in this study were $\mathrm{WBC}, \mathrm{RBC}, \mathrm{Hb}$, and PLT as routine blood tests; AST, ALT, and ALP for liver function; and UREA,
CRE, and UA for kidney function. The blood indicators of the subjects were divided into three levels: normal range, abnormalities with no clinical significance, and abnormalities with clinical significance. The results showed that the blood indicators of the two groups had no abnormal changes from baseline to posttreatment.

During the implementation of this study, one subject in the sham acupuncture group withdrew due to foot swelling. The research leader judged that the relationship with

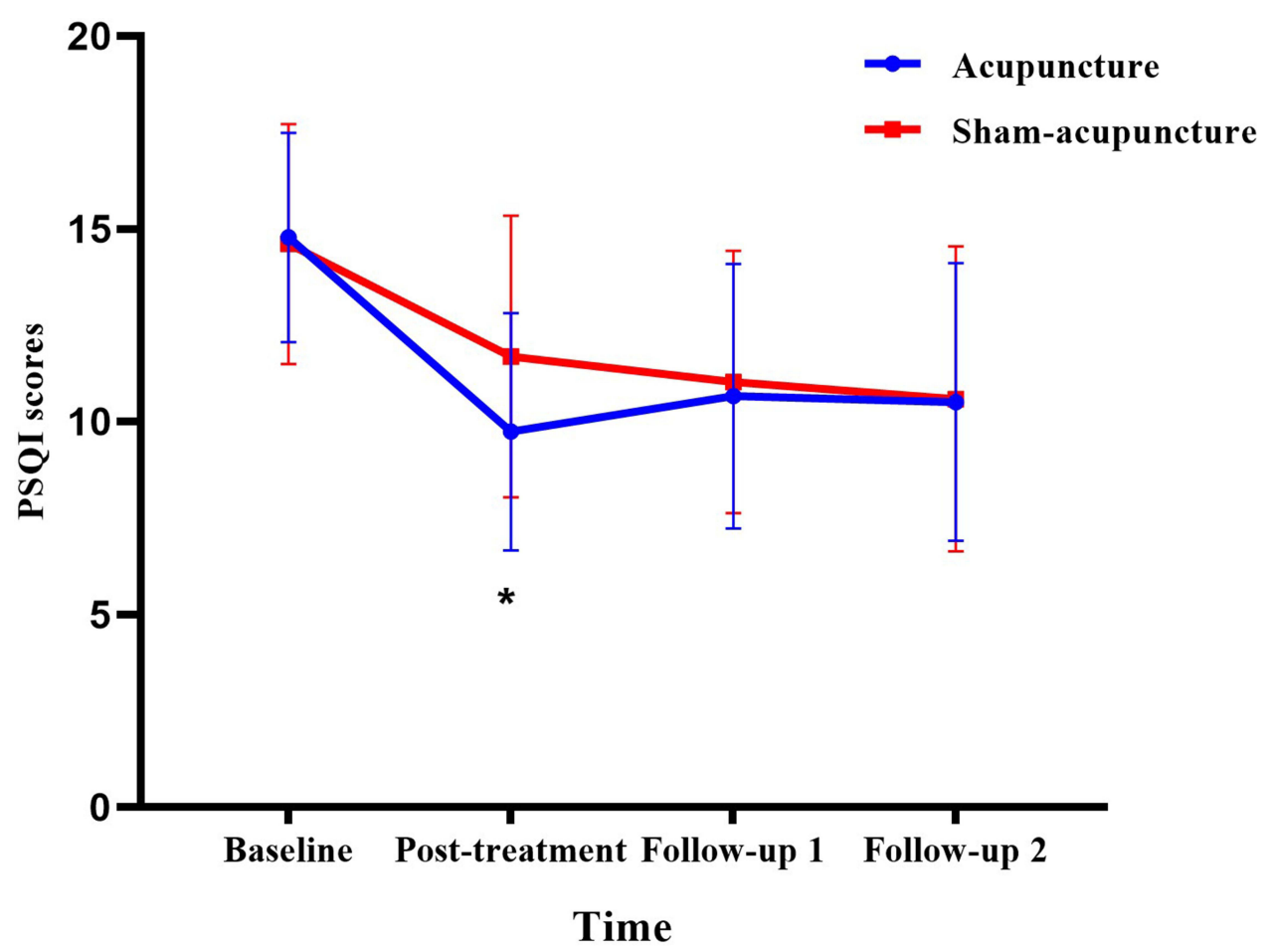

Figure 3 Mean PSQI scores of the two groups during the study period. Note: $* \mathrm{P}<0.05$ vs sham-acupuncture group. 


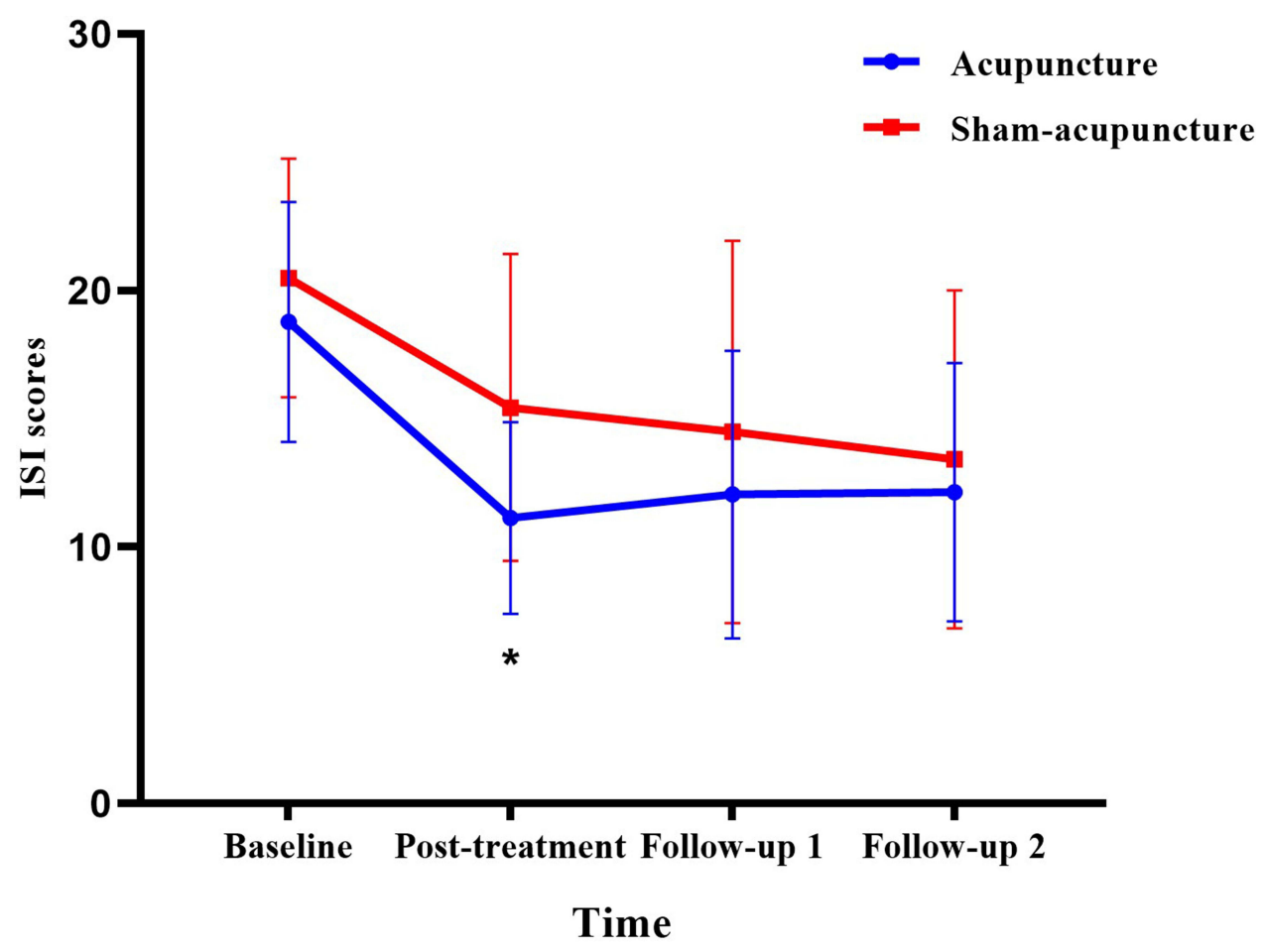

Figure 4 Mean ISI scores of the two groups during the study period. Note: ${ }^{*}<<0.05$ vs sham-acupuncture group.

intervention was "may not be relevant". Some subjects experienced local subcutaneous bleeding symptoms after the needles were removed, but the bleeding time did not exceed 1 minute, and there was no other discomfort. During the study period, no subjects experienced adverse reactions such as needle syncope, needle sticking, or subcutaneous haematoma, and no subjects experienced pain for longer than 30 minutes due to the intervention.

\section{Discussion}

This study is consistent with our team's previous research ${ }^{21}$ and other similar clinical studies of acupuncture for insomnia. ${ }^{18,71,72}$ The current study found that acupuncture can improve subjective and objective sleep indicators in patients with chronic insomnia. To the best of our knowledge, this is the first study to evaluate the efficacy and safety of using acupuncture at HT 7 and KI 7 to improve sleep and comorbid symptoms in chronic insomnia through subjective and objective measurements. On the basis of our previous research, ${ }^{21}$ we reduced the pain of subjects by streamlining the acupoints, improved compliance (drop-out rate $8.54 \%$ ), introduced more efficacy evaluation indicators, and extended the follow-ups to one month.
This study used the PSQI and ISI as the main outcome measures. We found that in the acupuncture group, PSQI and ISI scores decreased by 5.04 and 7.65 points, respectively, after 10 sessions of acupuncture treatment. Similarly, improvements were also found in other studies of acupuncture for insomnia. ${ }^{18,21}$ Previous research by our team has shown that for patients with perimenopausal insomnia, 10 sessions of acupuncture significantly reduced PSQI and ISI scores by 8.03 and 11.35 points, respectively. This study did not achieve the same effect. The reasons may be as follows: First, the acupoints were simplified in this study, and the effect of the treatment was reduced accordingly. Second, the previous study was aimed at people with perimenopausal insomnia, and appropriate acupoints can reduce perimenopausal symptoms and indirectly improve sleep symptoms. Third, compared with the previous study, the subjects recruited in this study had a lower severity of insomnia at the baseline analysis. In addition, there are some clinical studies on acupuncture for insomnia that have not achieved such a significant effect in subjective measurements. Xua Yin and colleagues ${ }^{18}$ found that the ISI score decreased 6.6 points after 4 weeks of acupuncture among subjects with insomnia. The study by Wing-Fai Yeung et $\mathrm{al}^{39}$ found no significant differences between groups in ISI or other outcome measures. The 
Table 4 PSG Measures from Baseline to Post-Treatment

\begin{tabular}{|c|c|c|c|c|c|c|c|c|}
\hline \multirow[t]{2}{*}{ PSG Assessments } & \multicolumn{3}{|c|}{ Acupuncture } & \multicolumn{3}{|c|}{ Sham-Acupuncture } & \multirow[b]{2}{*}{$Z / F^{b}$} & \multirow[b]{2}{*}{$P$} \\
\hline & $\bar{x} \pm s /$ M(Min, Max) & $Z / F^{a}$ & $P$ & $\bar{x} \pm s /$ M(Min, Max) & $Z / F^{a}$ & $P$ & & \\
\hline SOL(minutes) & - & \multirow{3}{*}{2.273} & \multirow{3}{*}{0.132} & - & \multirow{3}{*}{0.333} & \multirow{3}{*}{0.564} & & \\
\hline Baseline & $20.0(5.0,78.0)$ & & & $13.0(5.0,100.0)$ & & & $-1.14 \mid$ & 0.254 \\
\hline Post-treatment & $15.5(10.0,18.5)$ & & & I8.5(II.5, 3|.0) & & & -2.065 & 0.039 \\
\hline TST (minutes) & - & \multirow{3}{*}{7.364} & \multirow{3}{*}{0.007} & - & \multirow{3}{*}{8.333} & \multirow{3}{*}{0.004} & & \\
\hline Baseline & $384.5(226.5,452.5)$ & & & $353.0(214.5,443.0)$ & & & -1.016 & 0.310 \\
\hline Post-treatment & $404.0(318.5,458.0)$ & & & $394.0(219.0,475.0)$ & & & -1.046 & 0.295 \\
\hline WASO (minutes) & - & \multirow{3}{*}{7.364} & \multirow{3}{*}{0.007} & - & \multirow{3}{*}{5.333} & \multirow{3}{*}{0.021} & & \\
\hline Baseline & $74.5(\mid 3.0,242.5)$ & & & $84.5(12.0,259.0)$ & & & -1.037 & 0.300 \\
\hline Post-treatment & $41.5(9.5,109.0)$ & & & $76.25(1.5,258.0)$ & & & -1.416 & 0.157 \\
\hline SE (\%) & - & \multirow{3}{*}{2.836} & \multirow{3}{*}{0.018} & - & \multirow{3}{*}{3.399} & \multirow{3}{*}{0.006} & & \\
\hline Baseline & $73.66 \pm 13.63$ & & & $68.82 \pm 12.64$ & & & 1.011 & 0.321 \\
\hline Post-treatment & $80.93 \pm 9.25$ & & & $75.70 \pm 12.26$ & & & 1.147 & 0.264 \\
\hline Arl & - & \multirow{3}{*}{-1.789} & \multirow{3}{*}{0.104} & - & \multirow{3}{*}{1.157} & \multirow{3}{*}{0.272} & & \\
\hline Baseline & $17.38 \pm 8.96$ & & & $16.69 \pm 7.72$ & & & 0.224 & 0.824 \\
\hline Post-treatment & $|3.38 \pm 6.5|$ & & & $18.57 \pm 6.03$ & & & -1.984 & 0.060 \\
\hline NI (\%) & - & \multirow{3}{*}{-4.737} & \multirow{3}{*}{0.001} & - & \multirow{3}{*}{-1.735} & \multirow{3}{*}{0.111} & & \\
\hline Baseline & $31.61 \pm 10.38$ & & & $28.52 \pm 9.68$ & & & 0.844 & 0.406 \\
\hline Post-treatment & $19.65 \pm 5.06$ & & & $24.99 \pm 4.60$ & & & -2.654 & 0.015 \\
\hline N2 (\%) & - & \multirow{3}{*}{-1.919} & & - & & & & \\
\hline Baseline & $35.73 \pm 10.82$ & & 0.084 & $35.96 \pm 11.18$ & -1.695 & 0.118 & -0.056 & 0.955 \\
\hline Post-treatment & $29.13 \pm 9.03$ & & & $32.83 \pm 8.14$ & & & -1.035 & 0.312 \\
\hline N3 (\%) & - & & & - & & & & \\
\hline Baseline & $14.37 \pm 5.58$ & 6.846 & $<0.001$ & $17.55 \pm 8.34$ & 0.812 & 0.434 & -1.228 & 0.230 \\
\hline Post-treatment & $25.22 \pm 5.31$ & & & $17.88 \pm 4.84$ & & & 3.467 & 0.002 \\
\hline R (\%) & - & & & - & & & & \\
\hline Baseline & $18.30 \pm 5.50$ & 4.856 & 0.001 & $17.97 \pm 5.16$ & 4.407 & 0.001 & 0.168 & 0.868 \\
\hline Post-treatment & $26.01 \pm 3.30$ & & & $24.26 \pm 5.51$ & & & 0.914 & 0.371 \\
\hline
\end{tabular}

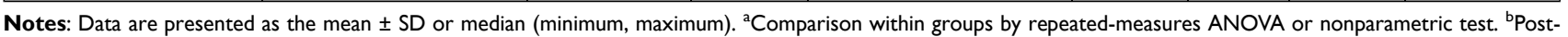
treatment comparison between groups by repeated-measures ANOVA or nonparametric test.

Abbreviations: PSG, polysomnography; SOL, sleep onset latency; TST, total sleep time; WASO, wake after sleep onset; SE, sleep efficiency; Arl, arousal index; R, rapid eye movement.

inconsistencies may be caused by differences in the choice of acupuncture points (number and attributes), acupuncture manipulation, and study populations. In this study, we provided direct evidence for the benefits of acupuncture at HT 7 and KI 7 in treating chronic insomnia among the Chinese population.

Among the factors that could affect acupuncture treatment for insomnia, the selection of acupoints is the most important. According to TCM theory, the selection for acupoints is based on three main strategies, ie, acupoints of the corresponding meridian, experience points, and special acupoint combinations. Therefore, each clinical study has different acupuncture point selections according to different
TCM theories. In previous studies, acupoints used for insomnia with high frequency were GV20 (Baihui), PC 6 (Neiguan), SP 6 (Sanyinjiao), HT 7 (Shenmen), GV24 (Shenting), and Ex-HN1 (Sishencong), ${ }^{41,73}$ which are experience acupoints for insomnia. Previous studies selected acupoints based on experience but lacked the guidance of TCM theory. We believe that these studies did not fully represent TCM acupoints. The disadvantage of empirical acupoints is that logic suggests that the more acupuncture points are selected for the treatment, the better the effect will be; however, that is not what is observed. Therefore, many studies have used a total of 10 or more acupoints according to the empirical acupoint principle, which not only fails to achieve 
Table 5 Comorbid Symptom Measurements from Baseline to Post-Treatment

\begin{tabular}{|c|c|c|c|c|c|c|c|c|}
\hline \multirow[t]{2}{*}{ Comorbid Assessments } & \multicolumn{3}{|c|}{ Acupuncture } & \multicolumn{3}{|c|}{ Sham-Acupuncture } & \multirow[b]{2}{*}{$Z / F^{b}$} & \multirow[b]{2}{*}{$\mathbf{P}$} \\
\hline & $\bar{x} \pm s /$ M(Min, Max) & $Z I^{a}$ & $\mathbf{P}$ & $\bar{x} \pm s /$ M(Min, Max) & $Z / F^{a}$ & $\mathbf{P}$ & & \\
\hline BAl score & - & & & - & & & & \\
\hline Baseline & $36(24,7 I)$ & 19.882 & $<0.001$ & $38(24,74)$ & 8.257 & 0.004 & -0.427 & 0.669 \\
\hline Post-treatment & $33(24,59)$ & & & $33(24,65)$ & & & -0.552 & 0.581 \\
\hline BDI score & - & & & - & & & & \\
\hline Baseline & $16(2,34)$ & 15.114 & $<0.001$ & $16(1,37)$ & 1.000 & 0.317 & -0.251 & 0.802 \\
\hline Post-treatment & II.5 (0, 39) & & & $13(0,31)$ & & & -1.031 & 0.303 \\
\hline FSS score & - & & & - & & & & \\
\hline Baseline & $40.59 \pm 14.46$ & 0.685 & 0.497 & $43.98 \pm 13.40$ & 1.414 & 0.166 & -1.101 & 0.274 \\
\hline Post-treatment & $38.13 \pm 13.65$ & & & $41.54 \pm 13.16$ & & & -1.115 & 0.269 \\
\hline ESS score & - & & & - & & & & \\
\hline Baseline & $9(0,19)$ & $<0.001$ & 1.000 & $10(0,21)$ & 1.400 & 0.237 & -0.019 & 0.985 \\
\hline Post-treatment & $8.5(1,24)$ & & & $6(0,18)$ & & & -1.729 & 0.084 \\
\hline
\end{tabular}

Notes: Data are presented as the mean \pm SD or median (minimum, maximum). ${ }^{a}$ Comparison within groups by repeated-measures ANOVA or nonparametric test. ${ }^{b}$ Posttreatment comparison between groups by repeated-measures ANOVA or nonparametric test.

Abbreviations: BAI, Beck anxiety inventory; BDI, Beck depression inventory; FSS, fatigue severity scale; ESS, Epworth sleepiness scale.

the expected effect, ${ }^{39}$ but also increases the subjects' suffering. Therefore, this study abandoned the principle of empirical acupoints and selected acupoints based on TCM and meridian theory. According to the theory of TCM, the comorbidity of the heart and kidney is an important factor in the occurrence of insomnia. Heart belongs to fire, and kidney belongs to water. The relationship between the heart and kidney is one of interaction and restriction. The rise of Yang in the kidney can warm the heart fire, and the heart fire can drop to the kidney, which can nourish the Yin in the kidney. If the functional relationship between the heart and kidney is imbalanced, pathological changes will occur. In addition to insomnia, the symptoms of this imbalance include anxiety, depression and other unhealthy emotions. This study used the theory of harmony between the heart and kidney to treat insomnia. The selected acupoints HT 7 and KI 7 belong to the Hand Shaoyin Heart Meridian and Foot Shaoyin Kidney Meridian, respectively, which satisfy the attributes of meridians and Zang-Fu in the treatment of insomnia according to TCM. The selected points on the meridians with the same name have an appropriate relationship for the mother-son reinforcing-reducing method. The results showed that acupuncture at HT 7 and KI 7 for insomnia had a benign effect based on the theory of harmony between the heart and kidney.

In terms of objective sleep measures, this study found that acupuncture can improve SOL and sleep architecture. Compared with the sham acupuncture group, the acupuncture group showed a statistically significant decrease in SOL. With respect to sleep architecture, less time in stage N1 and more time in stage N3 were found in the acupuncture group. Other studies on acupuncture for insomnia have rarely explored sleep architecture, although some have used PSG as an objective measure. Some similar studies suggest that acupuncture can improve sleep architecture by increasing the percentage of slow wave sleep (SWS), thereby improving sleep quality, ${ }^{74}$ which was consistent with our study. However, regarding the effects of acupuncture on sleep maintenance and SE, the results of our study are inconsistent with those of similar studies. Studies have suggested that acupuncture can improve TST and SE. ${ }^{18,21}$ This study found that although the acupuncture group showed increases in TST and SE, there was no significant difference between the groups. This may be due to the use of data from only the second consecutive night of PSG monitoring for statistical analysis in this study to exclude the "first night effect". Therefore, this result was more objective than previous studies, but due to the "first night effect", subjects might have been prone to compensatory sleep on the second night; that is, they may have slept better than usual. That is why the baseline analysis of the study showed that the mean TST was $>6$ hours, and the room for improvement was small. There are different conclusions about the impact of acupuncture for insomnia on objective sleep measures, and it is limited by many factors. 
In the future, acupuncture researchers may pay more attention to the recovery and stability of the sleep architecture.

It is worth noting that acupuncture could improve comorbid symptoms, but the difference was not statistically significant compared with sham acupuncture. The reasons may be as follows: First, this study only selected two acupoints (HT 7 and KI 7) to treat insomnia and comorbid symptoms. When selecting the acupoints, we mainly considered that HT 7 and KI 7 can improve insomnia symptoms. Although HT 7 has the effect of improving depression and anxiety symptoms and KI 7 has the effect of improving fatigue symptoms, the effect of a single acupoint may not be sufficient. Second, this study only adopted ten acupuncture treatments. However, chronic insomnia is a long-term disease. Our course of treatment may be relatively short and fail to achieve the expected clinical effect. Finally, this study mainly recruited subjects diagnosed with chronic insomnia and excluded patients with mental illnesses such as anxiety and depression disorders. Although the subjects generally had symptoms of anxiety and depression, they did not meet the relevant diagnostic criteria. Because of the "floor effect", the indicators improved, but the difference was not significant.

In addition, the results of this study showed that sham acupuncture had a benign effect on subjective and objective measurements. The results were similar to those of other clinical studies, suggesting that sham acupuncture has a certain clinical effect. ${ }^{39,40}$ A possible reason for this discrepancy may be that the participants we recruited had a positive attitude towards acupuncture treatment, and this high expectation might overoptimize the responses. The other reason may be that we used the true needle through, did not manipulate needles, and did not induce "De qi" but rather produced a shallow puncture through the skin. ${ }^{75}$ According to the theory of TCM, this sham acupuncture method belongs to the categories of "burr needling" and "shallow needling" in the Canon of Internal Medicine. It can exert curative effects by stimulating the twelve skin zones (superficial parts of human skin). In future research, we would expand the scope of recruitment and adopt more reasonable sham acupuncture methods.

Overall, acupuncture at HT 7 and KI 7 can significantly improve subjective and objective sleep indicators in subjects with chronic insomnia. While the subjects' anxiety and depression were reduced, no significant difference compared with sham acupuncture was observed. Acupuncture at HT 7 and KI 7 failed to significantly improve the subjects' daytime fatigue and sleepiness.
Acupuncture at HT 7 and KI 7 for insomnia had shortterm effects, and there was no significant difference between the groups during the follow-ups. This kind of acupuncture method has high safety and no obvious adverse effects and thus has value for further clinical exploration.

\section{Limitations}

This study has some limitations. First, the acupuncturist could not be blinded to the treatment group, and the measure of treatment fidelity was not established. Second, due to limited time and insufficient equipment, only some of the subjects were selected for PSG monitoring. The results may be not accurate. Finally, there was no difference between the two groups during the follow-up period. The precise effect duration of acupuncture at HT 7 and KI 7 needs more exploration.

\section{Conclusions}

The results of the current trial provide supportive evidence that acupuncture at HT 7 and KI 7 can be an effective, safe, and well-tolerated nonpharmacological intervention for insomnia using both subjective questionnaires and objective sleep measures. However, acupuncture at HT 7 and KI 7 can only provide a short-term effect, and a better combination of acupuncture points needs further exploration.

\section{Data Sharing Statement}

The data that support the findings of this study are available from the corresponding author (Yun-fei Chen) upon reasonable request. Additionally, the individual deidentified participant data are available after contacting the corresponding author via email (icyf1968@163.com). The data will be available immediately following publication without an end date.

\section{Acknowledgments}

The authors thank Xiao-peng Ma from Shanghai Research Institute of Acupuncture and Meridian for her comments and suggestions on the study. We also thank Pin-xian Huang from Shanghai University of Traditional Chinese Medicine for her guidance on statistical methods. We also thank Wen-jia Yang, Xin-tong $\mathrm{Yu}$ and $\mathrm{Na}$ Zhao from Yueyang Hospital of Integrated Traditional Chinese and Western Medicine, Shanghai University of Traditional Chinese Medicine. 


\section{Funding}

Shanghai Clinical Research Centre for Acupuncture and Moxibustion (Grant No. 20MC1920500); The National Natural Science Foundation of China (Grant No. 81904300); The Shanghai Science and Technology Commission (Grant No. 18401971000); The Construction of Chinese Medicine Heritage Innovation Platform (Grant No. ZY (2018-2020)-CCCX-2004-03); The Shanghai Municipal Commission of Health and Family Planning (Grant No. ZYKC201703006).

\section{Disclosure}

The authors report no conflicts of interest in this work.

\section{References}

1. Suh S, Cho N, Zhang J. Sex differences in insomnia: from epidemiology and etiology to intervention. Curr Psychiatry Rep. 2018;20 (9):69. doi:10.1007/s11920-018-0940-9

2. Chung KF, Yeung WF, Yu YM, Ho FY. A population-based 2-year longitudinal study of insomnia disorder in a Chinese population in Hong Kong. Psychol Health Med. 2018;23(5):505-510. doi:10.1080/ 13548506.2017.1363397

3. Brouwer A, van Raalte DH, Rutters F, et al. Sleep and HbA (1c) in patients with type 2 diabetes: which sleep characteristics matter most? Diabetes Care. 2020;43(1):235-243. doi:10.2337/dc19-0550

4. Cai G-H, Theorell-Haglöw J, Janson C, et al. Insomnia symptoms and sleep duration and their combined effects in relation to associations with obesity and central obesity. Sleep Med. 2018;46:81-87. doi:10.1016/j.sleep.2018.03.009

5. Tobaldini E, Fiorelli EM, Solbiati M, Costantino G, Nobili L, Montano N. Short sleep duration and cardiometabolic risk: from pathophysiology to clinical evidence. Nat Rev Cardiol. 2019;16 (4):213-224. doi:10.1038/s41569-018-0109-6

6. Li J, Huang Z, Hou J, et al. Sleep and CKD in Chinese adults: a Cross-Sectional Study. CJASN. 2017;12(6):885-892. doi:10.2215/ CJN.09270816

7. Hein M, Lanquart J-P, Loas G, Hubain P, Linkowski P. Objective sleep alterations and long-term use of short or intermediate half-life benzodiazepine receptor agonists are risk factors for high blood pressure in individuals with insomnia: a study in 1272 individuals referred for sleep examinations. Sleep Med. 2019;53:115-123. doi:10.1016/j.sleep.2018.08.030

8. Li L-J, Yang Y, Guan B-Y, et al. Insomnia is associated with increased mortality in patients with first-ever stroke: a 6-year follow-up in a Chinese cohort study. Stroke Vasc Neurol. 2018;3 (4):197-202. doi:10.1136/svn-2017-000136

9. Taylor DJ, Lichstein KL, Durrence HH, Reidel BW, Bush AJ. Epidemiology of insomnia, depression, and anxiety. Sleep. 2005;28 (11):1457-1464. doi:10.1093/sleep/28.11.1457

10. Taylor DJ, Mallory LJ, Lichstein KL, Durrence HH, Riedel BW, Bush AJ. Comorbidity of chronic insomnia with medical problems. Sleep. 2007;30(2):213-218. doi:10.1093/sleep/30.2.213

11. Francesmonneris A, Pincus H, First M. Diagnostic and Statistical Manual of Mental Disorders: DSM-V. American Psychiatric Association; 2013.

12. Riemann D, Baglioni C, Bassetti C, et al. European guideline for the diagnosis and treatment of insomnia. $J$ Sleep Res. 2017;26 (6):675-700. doi:10.1111/jsr.12594

13. Qaseem A, Kansagara D, Forciea MA, Cooke M, Denberg TD. Management of chronic insomnia disorder in adults: a clinical practice guideline from the American College of Physicians. Ann Intern Med. 2016;165(2):125-133. doi:10.7326/M15-2175
14. Krystal AD. The changing perspective on chronic insomnia management. J Clin Psychiatry. 2004;65(Suppl 8):20-25.

15. Buscemi N, Vandermeer B, Friesen C, et al. The efficacy and safety of drug treatments for chronic insomnia in adults: a meta-analysis of RCTs. J Gen Intern Med. 2007;22(9):1335-1350. doi:10.1007/ s11606-007-0251-z

16. Miyata A, Iwamoto K, Kawano N, et al. The effects of acute treatment with ramelteon, triazolam, and placebo on driving performance, cognitive function, and equilibrium function in healthy volunteers. Psychopharmacology. 2015;232(12):2127-2137. doi:10.1007/ s00213-014-3843-4

17. Liu S, Zhang B. Interpretation of "Guidelines for the diagnosis and treatment of insomnia disorder in China". Chin J Contemp Neurol Neurosurg. 2017. doi:10.3969/j.issn.1672-6731.2017.09.002

18. Yin $\mathrm{X}$, Gou M, Xu J, et al. Efficacy and safety of acupuncture treatment on primary insomnia: a randomized controlled trial. Sleep Med. 2017;37:193-200. doi:10.1016/j.sleep.2017.02.012

19. Sarris J, Byrne GJ. A systematic review of insomnia and complementary medicine. Sleep Med Rev. 2011;15(2):99-106. doi:10.1016/j. smrv.2010.04.001

20. Cheuk DKL, Yeung W-F, Chung KF, Wong V. Acupuncture for insomnia. Cochrane Database Syst Rev. 2012;9:CD005472. doi:10.1002/14651858.CD005472.pub3

21. Fu C, Zhao N, Liu Z, et al. Acupuncture improves peri-menopausal insomnia: a randomized controlled trial. Sleep. 2017;40(11). doi:10.1093/sleep/zsx153

22. Wu XF, Zheng XN, Wang Y, et al. [Acupuncture at acupoints along the meridians for primary insomnia: a multi-center randomized controlled trial]. Zhongguo Zhen Jiu. 2020;40(5):465-471. Chinese. doi:10.13703/j.0255-2930.20190430-k0001

23. Zhang L, Tang Y, Hui R, et al. The effects of active acupuncture and placebo acupuncture on insomnia patients: a randomized controlled trial. Psychol Health Med. 2020;25(10):1201-1215. doi:10.1080/ 13548506.2020.1738015

24. Xu F, Xuan LH, Zhou HJ, et al. Acupoint catgut embedding alleviates insomnia in different Chinese medicine syndrome types: a randomized controlled trial. Chin J Integr Med. 2019;25 (7):543-549. doi:10.1007/s11655-018-2770-3

25. Garland SN, Xie SX, DuHamel K, et al. Acupuncture versus cognitive behavioral therapy for insomnia in cancer survivors: a randomized clinical trial. J Natl Cancer Inst. 2019;111 (12):1323-1331. doi:10.1093/jnci/djz050

26. Chung KF, Yeung WF, Yu BY, et al. Acupuncture with or without combined auricular acupuncture for insomnia: a randomised, waitlist-controlled trial. Acupunct Med. 2018;36(1):2-13. doi:10.1136/acupmed-2017-011371

27. Foroughinia S, Hessami K, Asadi N, et al. Effect of acupuncture on pregnancy-related insomnia and melatonin: a single-blinded, randomized, placebo-controlled trial. Nat Sci Sleep. 2020;12:271-278. doi:10.2147/NSS.S247628

28. Buysse DJ, Ancoli-Israel S, Edinger JD, Lichstein KL, Morin CM. Recommendations for a standard research assessment of insomnia. Sleep. 2006;29(9):1155-1173. doi:10.1093/sleep/29.9.1155

29. Chen J, Theory Z. Traditional Chinese Medicine. Shanghai Medical University; 1985.

30. Feng S-W, Zeng F, Ren Y-L, Liang F-R. [Discussion on disease spectrum treated with acupuncture at shenmen (HT 7) and its compatibility based on bibliometrics]. Zhongguo Zhen Jiu. 2014;34 (6):561-564. Chinese.

31. Xie L, Yang R-D, Huang Q-L. [Rustic opinions on the theory of the therapeutic method of sweating disease by using Hegu (LI 4) and Fuliu (KI 7)]. Zhongguo Zhen Jiu. 2014;34(8):781-782. Chinese.

32. Schulz KF, Altman DG, Moher D; for the CONSORT Group. CONSORT 2010 Statement: updated guidelines for reporting parallel group randomised trials. BMJ. 2010;340(mar23 1):c332-c332. doi:10.1136/bmj.c332 
33. MacPherson H, Altman DG, Hammerschlag R, et al. Revised STandards for Reporting Interventions in Clinical Trials of Acupuncture (STRICTA): extending the CONSORT statement. $J$ Altern Complementary Med. 2010;16(10):ST-1-ST-14. doi:10.1089/acm.2010.1610

34. Sateia MJ. International classification of sleep disorders-third edition. Chest. 2014;146(5):1387-1394. doi:10.1378/chest.14-0970

35. Buysse DJ, Reynolds CF, Monk TH, Berman SR, Kupfer DJ. The Pittsburgh sleep quality index: a new instrument for psychiatric practice and research. Psychiatry Res. 1989;28(2):193-213. doi:10.1016/0165-1781(89)90047-4

36. Schutte-Rodin S, Broch L, Buysse D, Dorsey C, Sateia M. Clinical guideline for the evaluation and management of chronic insomnia in adults. J Clin Sleep Med. 2008;04(05):487-504. doi:10.5664/ jesm. 27286

37. Ibáñez V, Silva J, Cauli O. A survey on sleep questionnaires and diaries. Sleep Med. 2018;42:90-96. doi:10.1016/j.sleep.2017.08.026

38. Francesmonneris A, Pincus H, First M. Diagnostic and Statistical Manual of Mental Disorders: DSM-V. American Psychiatric Association; 2013. Available from: http://www.researchgate.net/pub lication/236017807_Diagnos. Accessed November 5, 2020.

39. Yeung W-F, Chung K-F, Zhang S-P, Yap T-G, Law ACK. Electroacupuncture for primary insomnia: a randomized controlled trial. Sleep. 2009;32(8):1039-1047. doi:10.1093/sleep/32.8.1039

40. Huang W, Kutner N, Bliwise DL. A systematic review of the effects of acupuncture in treating insomnia. Sleep Med Rev. 2009;13 (1):73-104. doi:10.1016/j.smrv.2008.04.002

41. Huang K, Liang S, Xu Y, Lu S. [Law of acupoint selection in acupuncture treatment for insomnia based on data mining method]. Zhongguo Zhen Jiu. 2015;35(9):960-963. Chinese.

42. White AR, Filshie J, Cummings TM. Clinical trials of acupuncture: consensus recommendations for optimal treatment, sham controls and blinding. Complement Ther Med. 2001;9(4):237-245. doi:10.1054/ ctim. 2001.0489

43. Zhou W, Benharash P. Significance of "Deqi" response in acupuncture treatment: myth or reality. J Acupunct Meridian Stud. 2014;7 (4):186-189. doi:10.1016/j.jams.2014.02.008

44. Zhao L, Li D, Zheng H, et al. Acupuncture as adjunctive therapy for chronic stable angina: a randomized clinical trial. JAMA Intern Med. 2019;179(10):1388. doi:10.1001/jamainternmed.2019.2407

45. Liu Z, Liu Y, Xu H, et al. Effect of electroacupuncture on urinary leakage among women with stress urinary incontinence: a randomized clinical trial. JAMA. 2017;317(24):2493. doi:10.1001/ jama.2017.7220

46. Brinkhaus B, Witt CM, Jena S, et al. Interventions and physician characteristics in a randomized multicenter trial of acupuncture in patients with low-back pain. J Altern Complementary Med. 2006;12 (7):649-657. doi:10.1089/acm.2006.12.649

47. Mollayeva T, Thurairajah P, Burton K, Mollayeva S, Shapiro CM, Colantonio A. The Pittsburgh sleep quality index as a screening tool for sleep dysfunction in clinical and non-clinical samples: a systematic review and meta-analysis. Sleep Med Rev. 2016;25:52-73. doi:10.1016/j.smrv.2015.01.009

48. Pilz LK, Keller LK, Lenssen D, Roenneberg T. Time to rethink sleep quality: PSQI scores reflect sleep quality on workdays. Sleep. 2018;41(5). doi:10.1093/sleep/zsy029

49. Backhaus J, Junghanns K, Broocks A, Riemann D, Hohagen F. Testretest reliability and validity of the Pittsburgh sleep quality index in primary insomnia. $J$ Psychosom Res. 2002;53(3):737-740. doi:10.1016/S0022-3999(02)00330-6

50. Zheng B, Li M, Wang KL, Lv J. [Analysis of the reliability and validity of the Chinese version of Pittsburgh sleep quality index among medical college students]. Beijing Da Xие Xиe Bao Yi Xиe Ban. 2016;48(3):424-428. Chinese.

51. Liu XC, Tang MQ. Reliability and validity of the Pittsburgh sleep quality index. Chin J Psychiatry. 1996;29:103-107.
52. Bastien C. Validation of the insomnia severity index as an outcome measure for insomnia research. Sleep Med. 2001;2(4):297-307. doi:10.1016/S1389-9457(00)00065-4

53. Chen P-Y, Yang C-M, Morin CM. Validating the cross-cultural factor structure and invariance property of the insomnia severity index: evidence based on ordinal EFA and CFA. Sleep Med. 2015;16 (5):598-603. doi:10.1016/j.sleep.2014.11.016

54. Morin CM, Belleville G, Bélanger L, Ivers H. The insomnia severity index: psychometric indicators to detect insomnia cases and evaluate treatment response. Sleep. 2011;34(5):601-608. doi:10.1093/sleep/ 34.5.601

55. Yu DSF. Insomnia severity index: psychometric properties with Chinese community-dwelling older people: insomnia severity index. J Adv Nurs. 2010;66(10):2350-2359. doi:10.1111/j.1365-2648.2010.05394.x

56. Miettinen T, Myllymaa K, Hukkanen T, Töyräs J, Sipilä K, Myllymaa S. Home polysomnography reveals a first-night effect in patients with low sleep bruxism activity. J Clin Sleep Med. 2018;14 (08):1377-1386. doi:10.5664/jcsm.7278

57. Berry RB, Brooks R, Gamaldo CE. The AASM Manual for the Scoring of Sleep and Associated Events (Version 2.3). American Academy of Sleep Medicine; 2017.

58. Beck AT, Epstein N, Brown G, Steer RA. An inventory for measuring clinical anxiety: psychometric properties. J Consult Clin Psychol. 1988;56(6):893-897. doi:10.1037/0022-006X.56.6.893

59. Kin-Wing SC, Chee-Wing W, Kit-Ching W, Heung-Chun GC. A study of psychometric properties, normative scores and factor structure of beck anxiety inventory Chinese version. Chin J Clin Psychol. 2002;10(1):4-6.

60. Liang Y, Wang L, Zhu J. Factor structure and psychometric properties of Chinese version of beck anxiety inventory in Chinese doctors. $J \quad$ Health Psychol. 2018;23(5):657-666. doi:10.1177/ 1359105316658971

61. Beck AT, Steer RA, Ball R, Ranieri WF. Comparison of beck depression inventories-IA and-II in psychiatric outpatients. J Pers Assess. 1996;67(3):588-597. doi:10.1207/s15327752jpa6703_13

62. Yeung A, Howarth S, Chan R, Sonawalla S, Nierenberg AA, Fava M. Use of the Chinese version of the beck depression inventory for screening depression in primary care. J Nerv Ment Dis. 2002;190 (2):94-99. doi:10.1097/00005053-200202000-00005

63. Wu P-C, Huang T-W. Gender-related invariance of the beck depression inventory II for Taiwanese adolescent samples. Assessment. 2014;21(2):218-226. doi:10.1177/1073191112441243

64. Krupp LB. The fatigue severity scale: application to patients with multiple sclerosis and systemic lupus erythematosus. Arch Neurol. 1989;46(10):1121. doi:10.1001/archneur.1989.00520460115022

65. Scullin MK, Bliwise DL. Sleep, cognition, and normal aging: integrating a half century of multidisciplinary research. Perspect Psychol Sci. 2015;10(1):97-137. doi:10.1177/1745691614556680

66. Wang M-Y, Liu I-C, Chiu C-H, Tsai P-S. Cultural adaptation and validation of the Chinese version of the fatigue severity scale in patients with major depressive disorder and nondepressive people. Qual Life Res. 2016;25(1):89-99. doi:10.1007/s11136-015-1056-x

67. Chervin RD. Epworth sleepiness scale? Sleep Med. 2003;4 (3):175-176. doi:10.1016/s1389-9457(03)00030-3

68. Omobomi O, Quan SF. A requiem for the clinical use of the Epworth sleepiness scale. J Clin Sleep Med. 2018;14(5):711-712. doi:10.5664/ jcsm.7086

69. Li-li PENG, Jin-rang LI, Jian-jun SUN, et al. Reliability and validity of the simplified Chinese version of Epworth sleepiness scale. Chin J Otorhinolaryngol Head Neck Surg. 2011;1:44-49.

70. Sakpal TV. Sample size estimation in clinical trial. Perspect Clin Res. 2010;1(2):67-69.

71. Guerreiro da Silva JB, Nakamura MU, Cordeiro JA, Kulay L. Acupuncture for insomnia in pregnancy - a Prospective, QuasiRandomised, Controlled Study. Acupunct Med. 2005;23(2):47-51. doi:10.1136/aim.23.2.47 
72. Zollman FS, Larson EB, Wasek-Throm LK, Cyborski CM, Bode RK. Acupuncture for treatment of insomnia in patients with traumatic brain injury: a Pilot Intervention Study. J Head Trauma Rehabil. 2012;27(2):135-142. doi:10.1097/HTR.0b013e3 182051397

73. Zhao K. Acupuncture for the treatment of insomnia. In: International Review of Neurobiology. Vol. 111. Elsevier;2013:217-234. doi:10.1016/B978-0-12-411545-3.00011-0
74. Hachul H, Garcia TKP, Maciel AL, Yagihara F, Tufik S, Bittencourt L. Acupuncture improves sleep in postmenopause in a randomized, double-blind, placebo-controlled study. Climacteric. 2012;16(1):36-40. doi:10.3109/13697137.2012.698432

75. Cummings M. Electroacupuncture is effective for control of myeloablative chemotherapy-induced emesis (N=104). Acupunct Med. 2001;19(1):54-69. doi:10.1136/aim.19.1.54

\section{Publish your work in this journal}

Nature and Science of Sleep is an international, peer-reviewed, open access journal covering all aspects of sleep science and sleep medicine, including the neurophysiology and functions of sleep, the genetics of sleep, sleep and society, biological rhythms, dreaming, sleep disorders and therapy, and strategies to optimize healthy sleep.
The manuscript management system is completely online and includes a very quick and fair peer-review system, which is all easy to use. Visit http://www.dovepress.com/testimonials.php to read real quotes from published authors. 